\title{
Contents
}

1. Ideas for moving beyond structure to dynamics of ecological networks

Daniel B. Stouffer, Miguel A. Fortuna, and Jordi Bascompte

1.1 Introduction . . . . . . . . . . . . . . . . 1

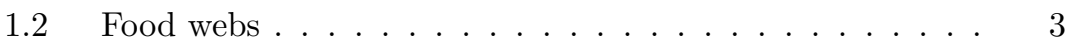

1.2.1 The structure of food webs . . . . . . . . . . 4

1.2.2 The scale of food-web stability . . . . . . . . . 5

1.2.3 Whole food-web dynamics ......... . . . 7

1.3 Mutualistic networks ............. 8

1.3.1 The structure of mutualistic networks . . . . . 9

1.3.2 Assembly of mutualistic networks . . . . . . . 11

1.3.3 Models of mutualistic-network disassembly . . . . 14

1.4 Spatial networks . . . . . . . . . . . . 15

1.4.1 The structure of spatial networks . . . . . . . 16

1.4.2 Unraveling the dynamics of spatial networks . . . 19

1.5 Concluding remarks .............. 21

$\begin{array}{ll}\text { Bibliography } & 23\end{array}$ 


\title{
Chapter 1
}

\section{Ideas for moving beyond structure to dynamics of ecological networks}

\author{
Daniel B. Stouffer, Miguel A. Fortuna, and Jordi Bascompte \\ Integrative Ecology Group \\ Estación Biológica de Doñana - CSIC \\ Av. de Maria Luisa s/n Pabellón del Perú \\ 41013 Sevilla (Spain)
}

\section{$1.1 \quad$ Introduction}

There are between seven and fifty million different species of plants and animals on Earth [Pimm and Raven (2000)]. About two-thirds of these species live in the tropics, largely in the tropical forests [Pimm and Raven (2000)]. In fact, studies show that about $30-50 \%$ of plant, amphibian, reptile, mammal, and bird species occur in 25 hotspots that occupy no more than $2 \%$ of the terrestrial land mass [Myers et al. (2000)]. It is believed that fish and other marine organisms are similarly concentrated [McAllister et al. (1994)].

The concentration of natural species demands that hotspots be managed with particular attention and caution [Ceballos et al. (2005); Ceballos and Ehrlich (2006); Hurlbert and Jetz (2007)]. Unfortunately only about one half of the original 16 million square kilometers of tropical rain forests remain [Skole and Tucker (1993)] and clearing eliminates about 0.2 million square kilometers every year [Nepstad et al. (1999); Cochrane et al. (1999); Hansen et al. (2008)]. This and other factors, such as human population growth and global warming, place us in the midst of the sixth largest extinction event in natural history [Thomas et al. (2004)].

The impacts are far reaching as extinctions of species represent one 
of the most dramatic ecosystem perturbations, taking place on quicker time scales than evolution and the introduction of new species into a habitat [Thomas et al. (2004)]. Extinctions have the ability to greatly alter an ecosystem's biodiversity; they can affect ecosystem stability, its resilience to environmental change, or its resistance to invasion of exotic species [Chapin et al. (2000)].

In the ocean, the story is no different. Currently $75 \%$ of global fish stocks are fully- or over-exploited [United Nations Food and Agriculture Organization (2006)]. Amongst these stocks are a considerable number of predators, such as sharks, which occupy the highest trophic levels; it is observed that these species have been declining at an alarming pace [Myers and Worm (2003); Worm et al. (2005); Heithaus et al. (2008)]. Moreover, recent empirical and theoretical studies have demonstrated that top predator removal often induces large-scale cascading effects [Bascompte et al. (2005)]. Many developed nations have made progress in better fishery management [Griffith (2008)]. However, nearly $20 \%$ of fish stocks in the United States, for example, remain over-fished or are fished unsustainably [National Marine Fishery Service (2008)]. The problem is more dire in developing countries, many of which rely upon fishing as an important economic activity [United Nations Food and Agriculture Organization (2006)].

There are numerous ecological, environmental, economic, and social aspects to the problems which stand before us. In order to remedy them, and avoid recreating such problems in the future, we must develop effective environmental policies which are firmly based on current environmental and ecological research. Reflecting upon the recent past and prospective future, research in the field of ecological networks is a strong candidate to successfully lead us in this direction.

In more traditional ecology, studies are restricted to an analysis of one to a few species within an ecosystem. In the study of food webs or mutualistic networks, in contrast, the focus is upon understanding the properties of the entire ecosystem [Bascompte et al. (2003); Pascual and Dunne (2006)]. Similarly, in the study of spatial networks, the focus is upon the application of the network formalism to problems in spatial ecology [Urban and Keitt (2001)].

The network approach in ecology has the longest tradition in community ecology and food webs, where it has been utilized over the last thirty years [Cohen (1978); Pimm (2002)]. Even so, the static and structural properties of food webs are only recently becoming better understood [Pascual and Dunne (2006)]. This leaves open a number of important questions 
regarding food-web dynamics and stability. Similar conclusions are reached when reviewing the mutualistic and spatial network literature.

If we wish to transform ecology, and in particular ecological networks, from a descriptive to predictive science, we must move beyond static characterization to the topic of dynamics upon these network structures. Consider, for example, the issue of overfishing. The effect of overfishing of a single species cannot be considered in isolation; it is imperative that the dynamics be viewed as a component of a far larger and more complex system. To effectively manage fisheries, then, we must understand the dynamics of the complete ecosystem and the influences of individual species upon all others. Similarly in spatial networks, we understand that species cannot be managed from the perspective of isolated local processes but through a combination of local and regional dynamics and exchanges across the system.

We will cover the topic of ecological networks in three separate sections which focus on the most developed sub-fields: food webs, mutualistic networks, and spatial networks. In this chapter, we authors shall not provide comprehensive reviews of previous work in the field of ecological networks. We will instead first briefly discuss the state of the field, emphasizing efforts and conclusions in understanding the systems' structural properties. We then discuss examples of investigations of the networks' dynamics, followed by what we believe to be some of the most exciting unanswered questions regarding the dynamic behavior, both for the near and far term.

\subsection{Food webs}

The tremendous diversity of ecosystems around the globe is apparent to even the most casual of observers. These ecosystems can differ in the population, sizes, and type of species present, the type of environment, the assembly history, and the rate of change. This diversity poses a very real challenge to the development of a general understanding of community dynamics.

Food webs are a description of who eats whom in an ecosystem [Sugihara (1984); Cohen et al. (1990)]. The food webs reported in the literature appear increasingly complex [Cohen et al. (1990); Pimm (2002); Pascual and Dunne (2006)]. Understanding the structure of food webs is of great importance because it provides insights into, for example, how ecosystems behave under perturbations [Berlow (1999); Chapin et al. (2000); McCann 
(A)

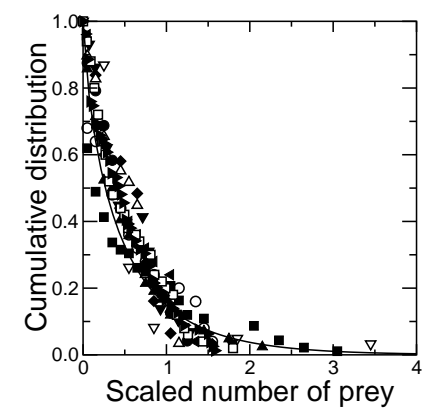

(B)

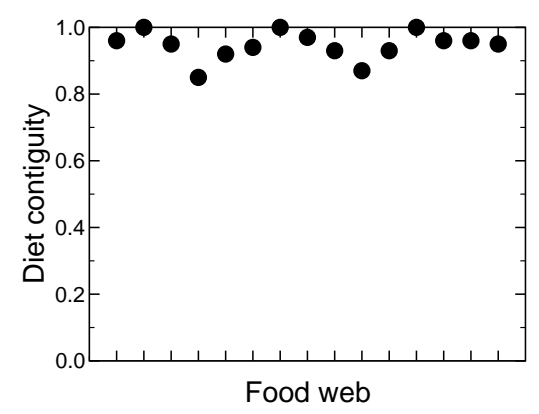

Fig. 1.1 Example of "universal" features of food web structure. (A), We plot the cumulative distribution of the scaled number of prey per species for 11 empirical food webs. The values for each web are scaled by twice the average number of predator-prey interactions per species in that web. The solid line is the analytical prediction for many successful static food-web models, including the niche model [Williams and Martinez (2000)]. The cumulative probability measures the fraction of events in a sample which have values larger than the set value. That the data "collapse" onto the same curve is a hallmark of universality. The same phenomenon is observed for the distributions of numbers of predators and links. (Adapted from Stouffer et al. (2005).) (B), We show the degree of diet contiguity for 14 empirical food webs, as measured by Stouffer et al. (2007). All 14 empirical food webs exhibit contiguity very close to 1.0, implying that species and their diets can very nearly be mapped onto a single dimension. This fact validates an important assumption of the niche model in which species and diets are explicitly one-dimensional.

(2000)].

\subsubsection{The structure of food webs}

Recent research on the structure of food webs has lead to a solution to the problem of developing a general understanding: there exist a number of universal features that hold for a large number of empirical food webs [Camacho et al. (2002); Dunne et al. (2002b); Cohen et al. (2003); Bascompte and Melián (2005); Stouffer et al. (2005); Pascual and Dunne (2006); Stouffer et al. (2006); Camacho et al. (2007); Stouffer et al. (2007); Allesina et al. (2008); Dunne et al. (2008); Petchey et al. (2008); Williams and Martinez (2008)]. These quantitative patterns describe the most highly-resolved empirical food webs available in the literature (Fig. 1.1). The most remarkable aspect of this result is that there were no a priori reasons to believe that the food webs studied have anything in common; in fact, the food webs studied have different sizes (ranging from 25 to 155 species), the species themselves are different, the empirical data was collected by different in- 
vestigators, and they come from a variety of environments located across the globe-lakes, streams, deserts, rain forests, estuaries, bays, and islands.

Relying upon this "universality" found in food webs, scientists have begun to reach a consensus on their static structural characteristics [Pascual and Dunne (2006); Williams and Martinez (2008); Allesina et al. (2008)]. These universal patterns demonstrate that there may indeed be fundamental principles which act as the determinants of food-web structure. Reinforcing this conclusion is a study by Dunne et al. (2008) which demonstrated that Cambrian food webs also exhibit the same structural properties. An explanation for this universality is the principle that there are emergent properties in complex systems which arise from constraints acting upon the system [Amaral and Ottino (2004)]. While bioenergetic constraints could be considered a major factor controlling food-web structure, it actually appears that the manner in which species select their prey may be a stronger driver force [Williams and Martinez (2000); Stouffer et al. (2006, 2007); Petchey et al. (2008); Williams and Martinez (2008)]. These robust patterns have led to the development [Williams and Martinez (2000)], and subsequent validations [Stouffer et al. (2005, 2006, 2007); Williams and Martinez (2008)] of a simple static food web model - the so-called niche model - that reproduces the key structural features observed in empirical food webs.

To this point, researchers who study food webs have followed a maxim observed in other disciplines, such as physics: one must understand statics before attempting to understand dynamics. As noted above, however, ecologists have begun to reach a consensus regarding the structure and mechanisms which shape the network of predator-prey interactions [Pascual and Dunne (2006); Stouffer et al. (2006); Camacho et al. (2007); Stouffer et al. (2007)]; therefore we believe that the time to advance our understanding of community-wide dynamics is at hand. In the following sub-sections, we will outline what we believe to be some of the most exciting and promising possibilities regarding the topic of food-web dynamics.

\subsubsection{The scale of food-web stability}

The complexity of empirical food webs has lead many ecologists to concentrate on dynamic ecosystem studies in terms of small sub-webs"community modules" [Holt (1997); Holt and Hochberg (2001)]—which bridge the gap between "the baroque complexity of entire communities and the bare bones of single and pair-wise population dynamics" [Holt (1997)]. 
(A)

Three species food-web motifs

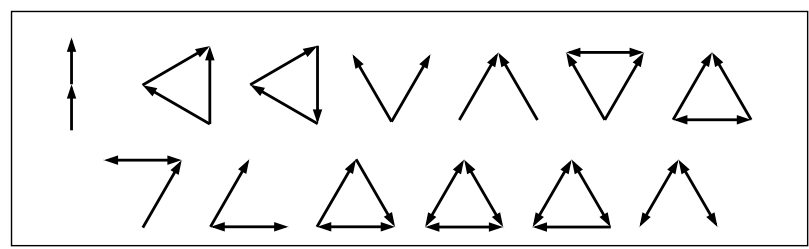

(B)
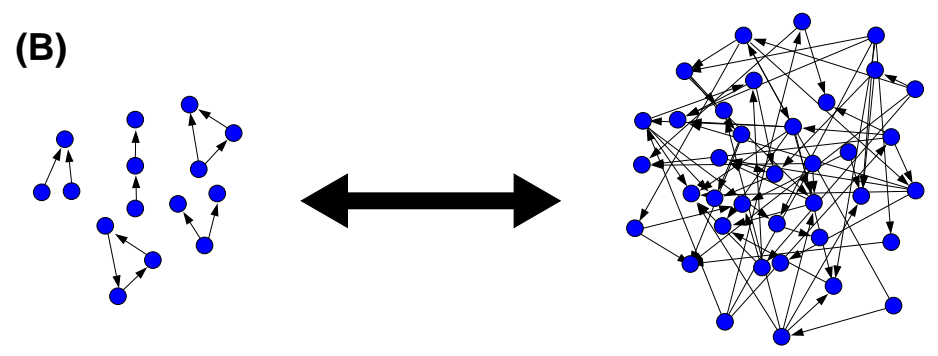

Fig. 1.2 Food-web motifs and the scale of food-web stability. (A), The set of 13 unique food-web motifs composed of three species [Milo et al. (2002); Stouffer et al. (2007)]. Each vertex represents a species and arrows represent predator-prey interactions pointing from prey to predator. (B), A significant unanswered question in the study of food webs is how the dynamic properties and stability of food-web motifs relates to those of the entire food web.

Community modules are comprised of three to five species and a set of interactions likely to have ecological relevance, such as a three-species food chain or intraguild predation.

The dynamic stability of community modules has been investigated previously; particular focus has been placed upon the implications of varying species interaction strengths and the role of weak interactions [McCann et al. (1998); Fussmann and Heber (2002); Emmerson and Yearsley (2004); Bascompte et al. (2005); Nakazawa and Yamamura (2006); Rooney et al. (2006)]. More recent research has also uncovered the important role of predator-prey body size ratios in stabilization of specific community modules such as a tri-trophic food chain [Otto et al. (2007)]. These bottom-up studies provide a theoretical foundation for the stability of individual modules, but leave open the question of which modules empirical food webs are actually composed of. To address this question, scientists have investigated "food-web motifs", the structural counter-part to community modules [Milo et al. (2002); Bascompte and Melián (2005); Stouffer et al. 
(2007)]. Food-web motifs consist of the complete set of unique connected subgraphs containing $n$-species [Milo et al. (2002)] (Fig. 1.2). These topdown studies provide an indication of the subgraphs which appear within empirical food webs more or less often than expected at random. They are unable, however, to address if there is a dynamic justification for why a particular subgraph appears with greater or less frequency.

It thusly follows that a significant unanswered question in the study of food-webs is the integration of the bottom-up and top-down approaches to better understand food-web dynamics and the origins of food-web stability. Is there a direct relationship between the "local" stability of a subgraph and on whether or not that subgraph appears more or less frequently in a complete food web? If no direct relationship exists, it is worth noting that this does not preclude the existence of a relationship at a "mesoscale" level of combinations of motifs.

The identification of each modules' contribution to community stability will provide crucial information regarding the forces acting upon the ecosystem. We will be able to answer an important question regarding the mechanisms responsible for maintaining stability in the presence of external perturbations. Namely, is a community stable because it is composed of stable sub-elements, or is a community stable because of cooperative and synergistic interactions between individual, and potentially unstable, sub-elements? Do species participate in interactions which would maximize their own persistence or instead that of the entire community?

\subsubsection{Whole food-web dynamics}

An ever-present problem in the study of empirical food webs is the relative scarcity of empirical data. Much of this stems directly from the difficulties involved in collecting data of high quality [Paine (1988)]. These difficulties including the long hours required for direct observation and for data collection to conduct stomach content analysis or scatology. Additionally, it is still an issue to know when a scientist may faithfully declare any data as complete; how does one account for the brief and sole appearance of a migratory bird, for example, within the environment under investigation? Consider also that these difficulties are faced when the food web will ultimately represent an aggregate over time and space [Lawton (1989)] and therefore the result is generally absent of dynamic data.

To date, the most frequently utilized approach to quantitatively measure a dynamic food-web property is through the interaction strength [Berlow 
et al. (2004)]. Most commonly this is represented as the fraction of a species' diet or incoming biomass which comes from particular prey; however, many definitions exist Berlow et al. (2004). While such interaction strengths are informative and can even help parametrize dynamics models [Yodzis and Innes (1992); Brose et al. (2006)], they themselves often represent averages across time and space. This is because of the fact that ecosystem dynamics necessarily implies dynamics of all aspects of the food web, from abundances to species' interaction strengths.

For future characterization and understanding of community dynamics, it is essential that we support initiatives for the collection of dynamic data for entire ecosystems, potentially even to the scale of the Long Term Ecological Research Network (LTER) or National Ecological Observatory Network (NEON) programs in the United States. Such initiatives, however, will represent decades of coordinated work with the majority of benefits realized far into the future.

A different possibility is to approach the problem from the bottomup instead of top-down. There exist databases (e.g., NERC Centre for Population Biology (1999)) which have compiled species-specific dynamic data. However, as in the case off food-web stability discussed earlier, it is not readily apparent how laboratory or field experiments of three species, for example, might translate to dynamics of greater collections of species.

One should consider that the species-specific data often represent the dynamics of individual species not in isolation but actually embedded within larger communities. We must first develop a robust and general characterization of the dynamic behaviors exhibited in this species-specific data. This could be conducted through a mixture of data analysis and modeling. Then the dynamics of species within food-web models should be similarly characterized for comparison purposes. These two characterizations will greatly help the refining of dynamic food-web models by determining cause of differences between dynamics in isolation or within an entire food web.

\subsection{Mutualistic networks}

To this point, we have defined the broad type of food web, which is the type of network with a longest tradition in ecological research. In the last few years, there has been a tendency to consider other types of interactions besides the strict who-eats-whom. The interactions between hosts and their animal parasitoids (i.e., insects that lay their eggs near, inside, or on the 


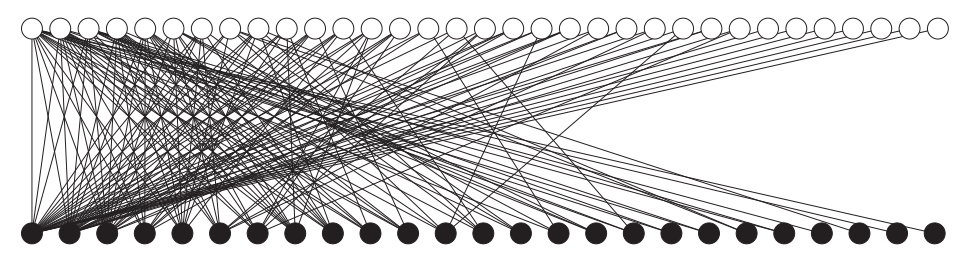

Fig. 1.3 Example of a bipartite plant-animal mutualistic network. Solid and empty nodes represent plant species, and their seed dispersers, respectively. These mutually beneficial interactions form networks of dependence involving dozens of species.

surface of the insect host which will provide food for the developing pupa) is a good example. A second type of interaction is that between plants and their specific herbivores, or the one between plants and the animals that pollinate their flowers or disperse their seeds. In all cases, we are now considering networks represented as bipartite networks (Fig. 1.3). In this section, we will focus on one type, that which describes the mutualistic interactions between plants and animals. There are several reasons for this. On one hand, these interactions of mutual benefit have played a major role in the generation of Earth's biodiversity [Ehrlich and Raven (1964); Thompson (2005)]. On the other hand, ecologists have compiled an impressive data set amenable to analysis by tools from study of complex networks.

Mutualistic interactions involve dozens to hundreds of species and form complex networks (Fig. 1.3). This is a complicated object, and one may be tempted to conclude that there is no pattern beyond an ocean of links and nodes. The earliest work on mutualisms was focusing on a highly specific plant-animal interaction, or on a few interactions among a target group of species. In the last few years, however, ecologists and evolutionary biologists acknowledged the community context of these interactions and worked on small groups of interacting species [Waser et al. (1996); Thompson (2005)]. More recently, these studies have been extended by scaling all the way up from small to entire networks and by providing a rational framework to characterize their complexity.

\subsubsection{The structure of mutualistic networks}

The first round of papers on mutualistic networks were, not surprisingly, descriptions of their structure, following the tradition in food webs as de- 
(A)

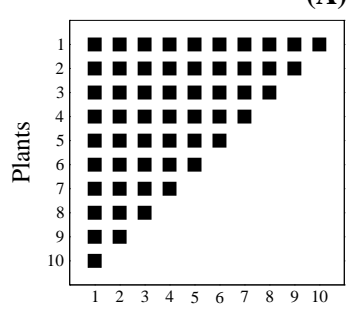

Animals

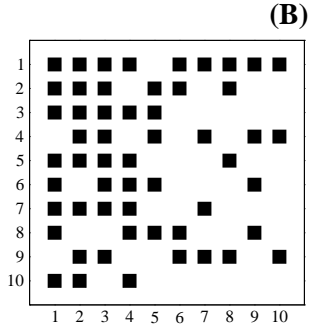

Animals

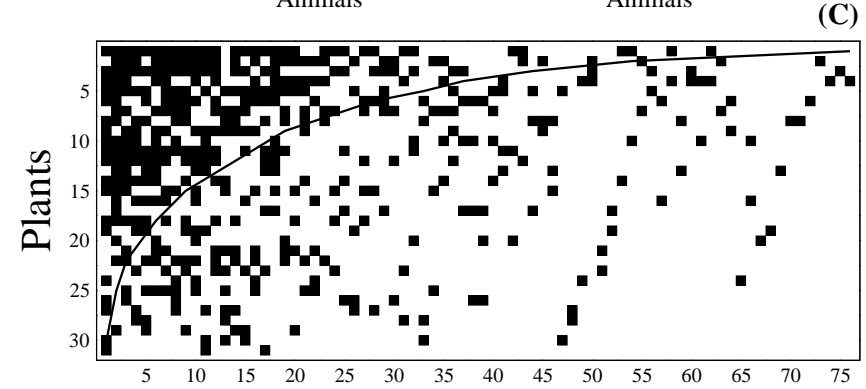

Animals

Fig. 1.4 The nested structure of mutualistic networks. The mutualistic network is now represented in matrix format, where rows represent plants, columns represent animals, and a square indicates an interaction between that plant and animal. (A) represents a perfectly nested matrix, where specialist species interact with well-defined subsets of the species generalists interact with, (B) represents a similar matrix with interactions randomly shuffled, and (C) shows a real mutualistic network where the continuous line indicates the isocline of perfect nestedness. (Figure modified from Bascompte et al. (2003).)

scribed in the previous section. The first pattern analyzed was the connectivity distribution, and ecologists found a common structural pattern defined by truncated power-law connectivity distributions. This put mutualistic networks in a similar context as other networks in the sense of a heterogeneous distribution. While the bulk of species had only one or a few interactions with other species, a few generalists had far more interactions than would be expected under conventional models [Jordano et al. (2003)]. Regardless of the type of mutualism (seed dispersal or pollination) and other biological differences, all communities had the same structure. A small difference, in relation to non-biological networks such as the Internet, was the truncation for high numbers of interactions. This truncation can be explained by several non-exclusive mechanisms such as forbidden links - some interactions between a plant and an animal can not occur due 
to, for example, size or phenological constraints [Jordano et al. (2003)] —or a preferential attachment mechanism on a bipartite network with different set sizes [Guimarães et al. (2007)].

Mutualistic networks are, thus, heterogeneous. However, this property only refers to a statistical description of the probability to interact with a given number of species. It does not tell us anything about the identity of the interacting species. The next step in the road to disentangle the structure of mutualistic networks was describing its nested structure (Fig. 1.4). In a nested matrix, the number of interactions per species are arranged in such a way that specialists interact with proper subsets of the species with which generalists interact [Bascompte et al. (2003)]. This generates a network with a core of interactions, generalist plants and generalist animals interacting among themselves, and asymmetric tails, specialists interacting with the most generalist species.

This asymmetry in specialization, independently reported by Vázquez and Aizen (2004), is also evident when looking at weighted networks. The weight of a link indicates the intensity of the mutualistic effect or dependence of one species on another. The bulk of dependencies between species are quite weak, but a few are strong. In the few cases in which, for example, a plant depends strongly on an animal, the animal depends on the plant very weakly, and vice versa [Bascompte et al. (2006)]. That is, pairwise interactions are very asymmetric. Similarly, one can also look at the quantitative extension of species degree, species strength. The strength of a plant species, for example, is the sum of all dependencies of the animals which depend on that plant. It describes the importance of a species from the point of view of those in the other set. Species strength varies widely across species [Bascompte et al. (2006)]. As for binary data, mutualistic networks are also very heterogeneous in the distribution of species strength.

\subsubsection{Assembly of mutualistic networks}

So far, we have provided a descriptive account of mutualistic networks. The dynamics, as always, are more complicated to tackle from the experimental point of view. There are a few exceptions from the literature, however, that provide a link from structure to dynamics. Olesen et al. (2008) analyzed the day-to-day assembly of one pollination network in Zackenberg field station, Greenland. This is a particularly convenient system since the whole area is covered by ice the bulk of the year. This implies that from the beginning of each season, one can record the entire network assembly from 
the appearance of the first flower, the first pollinator, and so on.

One example of a connection between network structure and dynamics was the pioneering paper by Barabasi and Albert (1999) on the preferentialattachment mechanism as a simple process leading to a scale-free network. In this case, one starts with a core of interacting nodes; at each time step, a new node enters the network and attaches to an existing node with probability proportional to the existing node's number of links. This process results in a network with a scale-free degree distribution [Barabasi and Albert (1999)]. It is one thing, however, to theoretically show that a process leads to a particular statistical pattern and quite another to demonstrate that an empirical system truly behaves in such a manner. To elaborate, upon finding a power-law connectivity distribution it may be tempting to assume a preferential-attachment process. Similar difficulties have permeated the study of other complex systems when scientists were tempted to conclude the existence of self-organized criticality from the presence of a power-law in the frequency distribution of events with a given size or energy.

The Zackenberg pollination network provided a wonderful opportunity to test the true dynamical assembly mechanism in the field. The temporal sequence for the whole season can be broken into day-to-day intervals so that, when a new interaction is formed, one can identify the precise identity of the species a new one attaches to and then test relative to the likelihood that a species would attach preferentially to the most connected species (Fig. 1.5). Olesen et al. (2008) found that attachment is intermediate between preferential and random. Intriguingly, this dynamic result is compatible with the previously reported pattern of a truncated power-law connectivity distribution.

Despite the previous result on actual processes of assembly, we are missing information on the dynamics of mutualistic networks, i.e., on how they change through years (see however Petanidou et al. (2008) and Olesen et al. (2008) for interesting exceptions). The most natural way to proceed due to the scarcity of dynamic data, is to use numerical simulations or mathematical models of network dynamics. A few papers have addressed models of network build-up which are directly comparable to the actual assembly in Zackenberg. For example, defining a preferential-attachment model on a bipartite network has provided an analogy to the Barabási-Albert model for unipartite networks [Guimarães et al. (2007)].

Interestingly enough, the nature of these bipartite networks imposes itself some changes in the resulting connectivity distribution when there is some difference in size between the two types of nodes. In this case, a 


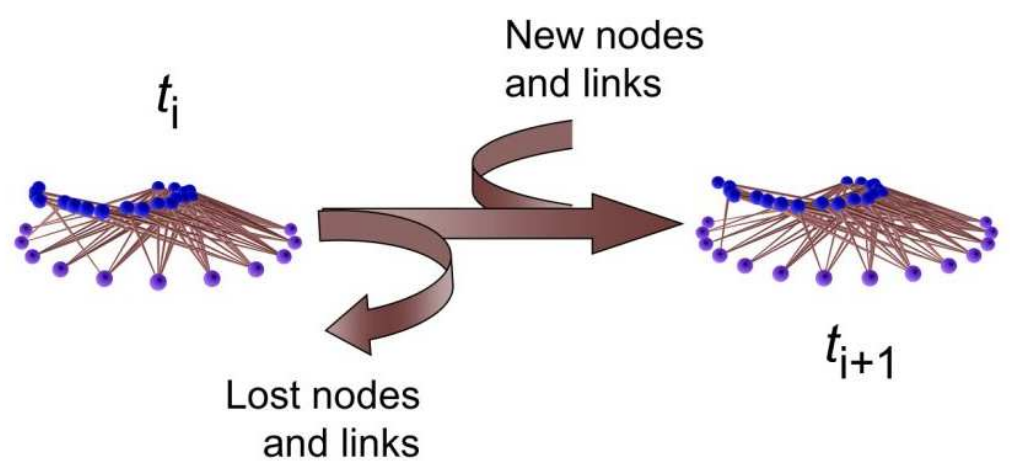

Fig. 1.5 The dynamics of network assembly. The figure illustrates the turnover in number of nodes and links between two consecutive days. Knowing whether new species tend to become attached to the already well-connected species is important in bridging structural patterns of mutualistic networks to their underlying assembly dynamics. (Figure courtesy of J. Olesen.)

truncated power-law connectivity distribution (as opposed to a power-law) naturally arises. Similarly, Santamaría and Rodríguez-Gironés (2007) have explored a suite of assembly models, concluding that the best fit to the structure of mutualistic networks is performed by a model that combined species abundance and phenotypic complementarity between morphological traits. In line with this previous result, phenotypic complementarity and the hierarchical evolutionary relationships between species have also been adduced to be a good explanation for the values of nestedness observed in nature [Rezende et al. (2007a)]. This is in agreement with conclusions from observations from the field [Stang et al. (2007)]. A combination of species abundance and niche-traits (e.g., morphological constraints) seems also to account for the levels of nestedness observed in nature [Krishna et al. (2008)].

We need general models that account not only for a network property such as nestedness but for a broad combination of network patterns, or, as Allesina et al. (2008) have recently put it, for the likelihood of reproducing an entire mutualistic network. We believe it is quite feasible to soon have such a generalized model of assembly of mutualistic networks which is able to reproduce the full range of network patterns discovered in the initial studies of mutualistic networks. 


\subsubsection{Models of mutualistic-network disassembly}

A more difficult challenge - and a much more interesting question, as tends to be the case - would be to develop an experimental setting to examine the disassembly of mutualistic networks as a function of some external driver. This is quite difficult to realize in the field, and even in a mesocosm experiment, yet is probably the most relevant question from the point of view of exploring the consequences of global change on biodiversity. The most exciting possibility would be an experimental system where one could simulate the effects of species extinction or habitat alteration on the network of interactions. In the meantime, we again must rely on numerical simulations. In the most common methodology a progressive number of species goes extinct and the influence of network structure on the size of the coextinction cascade, or the subsequent loss of evolutionary history, is investigated. The consensus result, as shown by Memmott et al. (2004) building on the papers by Albert et al. (2000) for the Internet and Solé and Montoya (2001) and Dunne et al. (2002a) for food webs, is that the heterogeneous, nested, structure of mutualistic networks makes them robust to the loss of specialists or to the random loss of species [Memmott et al. (2004)].

When one examines, however, the identities of the coextinct species and their phylogenetic positions, there is a significant phylogenetic signal on both the number of interactions per species and on whom they interact with [Rezende et al. (2007b)]; this is true for as many as half of the communities examined. Because of this, coextinction cascades do not involve randomly picked species but phylogenetically close species. The loss of evolutionary history then proceeds faster than expected in the absence of such phylogenetic signal, leading to a biased pruning of the evolutionary tree [Rezende et al. (2007b)].

Another driver of global change is habitat loss. There is only one study we are aware of that empirically studies the consequences of habitat transformation on network structure. Tylianakis et al. (2007) explored how the structure of host-parasitoid networks changes across an environmental gradient. They demonstrated that even without a reduction in the number of species, habitat loss changes the structure of interaction networks with important implications for their collapse. To further assess the influence of habitat transformation, requires the use of models.

An important area of research is the study of metacommunities, species interactions across discrete habitat patches maintained by a balance be- 
tween local processes and regional dispersal across patches [Leibold et al. (2004); Holyoak et al. (2005)]. This important area has mainly focused on theoretical work consisting of a small number of interacting species. Melián et al. (2005) and Fortuna and Bascompte (2006), on the other hand, have studied real ecological networks across space from a theoretical perspective but with added realism by using the structure of the real ecological networks.

A first step towards understanding the consequences of habitat loss on mutualistic networks was the study of a spatially implicit model of metacommunities in which two real mutualistic networks were used as a skeleton for the theoretical model [Fortuna and Bascompte (2006)]. The heterogeneous, nested, structure of mutualistic networks confers a higher level of robustness to habitat loss. While species start to go extinct sooner than for expected at random, the network as a whole persists for greater values of habitat loss [Fortuna and Bascompte (2006)]. This is only a first step, however, because the metacommunity is assumed to live in an idealized spatially implicit model composed by an infinite number of patches with similar dispersal to any other patch. It maintains the structure of the mutualistic network but not the structure of the habitat landscape. We anticipate that the tendency to study full ecological networks on real ecological landscapes will be an important area in the near future. This will integrate networks of networks. The spatial component of networks has already generated important contributions which we will review in the following section. A next step will be to integrate all sections, that is, to study the spatial component of networks of ecological interactions.

\subsection{Spatial networks}

We have thus far examined where we are in bridging the gap between structural understanding and system dynamics in food webs and plant-animal mutualistic networks. Such ecological networks depict relationships which principally affect the population growth rates of the interacting species. An altogether different type of ecological network, that representing spatial dynamics, has been also studied. Spatial dynamics influences both the organization and stability of communities [Tilman and Kareiva (1997); Hanski and Gilpin (1997); Bascompte and Solé (1998)] and hence, the spatial aspects in which ecological processes take place cannot be ignored. For example, in fragmented landscapes, the spatial distribution of habitat patches 
can influence on the dispersal movements of individuals [Wiens (2001)]. In plants, gene flow mediated by animals is a key demographic process which serves to shape the spatial pattern of intraspecific genetic variability [Barrett and Harder (1996)]. In animal societies, the relationships between individuals are often determined by the common use of resources patchily distributed across the landscape [Gibbons and Lindenmayer (2002)]. All these cases can be described and analyzed as networks in which nodes represent habitat resources and links indicate dispersal, gene flow, or social interactions. By consider spatial dynamics from a network perspective, we can shed light into problems as diverse as species persistence in fluctuating environments and information exchange between individuals in an animal society.

\subsubsection{The structure of spatial networks}

The spatial dynamics which result from the dispersal of individuals from one population to another has been successfully studied using the metapopulation framework [Hanski and Gilpin (1997)]. In this approach, the probability for an empty habitat patch to be colonized depends, among other factors, on the distance between that patch and the rest of occupied patches [Ovaskainen and Hanski (2004)]. By considering different functional forms for the probability of an individual to reach a patch located at a particular distance (i.e., the dispersal kernel), we can build stochastic networks of connected patches in fragmented landscapes. The structure of the resulting networks of patches provides a straightforward way to quantify the robustness of a patchy population to habitat loss [Urban and Keitt (2001)]. It also provides useful information for conservation planning because it allows the identification of the most important patches - termed keystone patches - that are critical for landscape connectivity and hence population persistence [Urban and Keitt (2001); Minor and Urban (2008)].

There are a few key examples of how the spatial dynamics resulting from individual movements between habitat sites shapes the structure of a spatial network of patches. Fortuna et al. (2006) showed that the structure of a large network of temporary ponds - used as breeding sites by several amphibian species - changes with increasing levels of drought (Fig. 1.6A). When drought was very intense and the number of dry ponds increased, only a few flooded ponds were accessible from a high number of dry ones. In this way, a ranking of ponds was established as a function of their connections to dry ponds, constrained by the dispersal abilities of species. In 

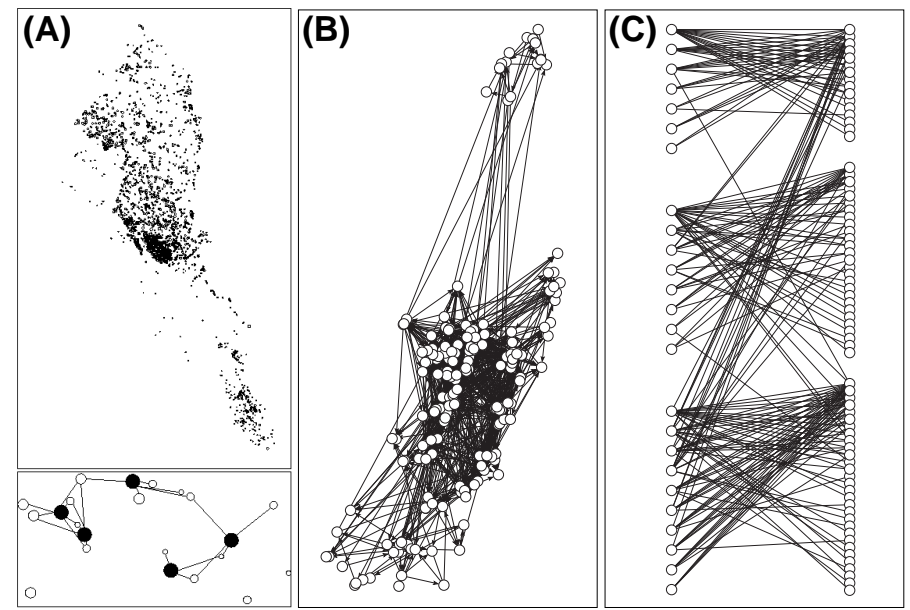

Fig. 1.6 Examples of spatial networks. (A), Spatial location of more than 3000 ponds in Doñana National Park (Spain). Below, schematic representation of a subset of ponds showing links from dry (white circles) to flooded (black circles) ponds mediated by the maximum dispersal distances of an amphibian species. (B), The mating network of a Prunus mahaleb population. Nodes represent trees and arrows indicate pollination events mediated by insects. (C), The bipartite roosting network of a bird-predator bat. On the left, nodes represent individual bats. On the right, nodes represent individual trees. A link between a bat and a tree indicates that that particular bat used than particular tree. (Adapted from Fortuna et al. $(2006,2008 \mathrm{a}, \mathrm{b})$.)

similar systems, such as riverine networks, the fragmentation over time of network of populations of a fish species was investigated and used to propose mechanisms for ecologically successful restoration [Schick and Lindley (2007)].

The ecological and evolutionary processes shaping the spatial distribution of genetic variability include natural selection, genetic drift, mutation, non-random mating, and gene flow by migration, to name a few. The cessation of gene movement because of limited dispersal or assortative mating results in genetic isolation and allows drift and other microevolutionary processes to differentiate non-connected populations. This in turn can lead to speciation. Understanding the spatial structure of the genetic variation is therefore a major goal for conservation and evolutionary biology because it contributes to the management of threatened populations and, ultimately, can lead to evolutionary change. 
To this end, molecular markers provide invaluable information about gene flow at individual and population levels. It is now possible to trace back pollen movement between the mother and the siring tree using paternity assignment techniques on collected seeds. One can then calculate the distance traveled by pollen carried by the wind or mediated by insects. With sufficient seed collection and by knowing the precise identity and location of all the trees of a population, we can build the mating network. In the mating network, nodes represent trees and links indicate the pollen movement from donor to mother tree (Fig 1.6B). Fortuna et al. (2008a) have shown that there exists a non-random pattern of pollen movement in an insect-pollinated tree. The population was structured in well-defined compartments formed by groups of mother trees and their shared pollen donors. They also found that the few long-distance pollination events reduced the compartmentalized structure of the mating network, increasing gene flow and hence reducing the role of genetic drift. As we will see later, there is a huge potential in the application of the complex network framework to gene-flow and population genetics.

Some species depend on resources patchily distributed across the landscape for shelter, such as hollows in trees [Gibbons and Lindenmayer (2002)]; bat colonies are a good example. Some bat species constitute fission-fusion societies whose members spread every day in multiple trees for shelter. The regular roost-switching movements of animals can be considered as channels that transport information or parasites among individuals. What structural patterns emerge from this spatial dynamics? In public parklands, the old trees used for shelter by bats are in danger of being removed by management agencies because of the potential danger to people from falling branches. In this case, the identification of the most important roosting locations would favor more efficient management solutions.

Using information on radio-tracked bats, Fortuna et al. (2008b) built a bipartite network establishing links between bats and trees when a particular bat used a particular tree (Fig. 1.6C). They observed the existence of well-defined colonies of bats, a compartmentalized structure similar to that found for the mating network of plants. Moreover, when the pattern of individual use of each tree inside each colony was examined, a nested network structure was detected. That is, as observed in mutualistic networks, bats using a few roosting trees are a subset of the bats that use trees used by a high number of bats. In the same way, trees that are used by a few bats are a subset of the trees used by bats that use many trees [Fortuna et al. (2008b)]. 
We have seen how the network approach can be successfully applied to characterize structural patterns emerging from spatial dynamics in ecology. What can the topological properties of these networks tell us about the dynamics of spatial processes?

\subsubsection{Unraveling the dynamics of spatial networks}

Following with the last case study, the spatial structure of the roosting network can provide insights into the functionality of roost changes and social grouping or segregation by describing the way that information, diseases, or parasites can travel through the network. The compartmentalized structure implies that each colony of bats uses a subset of trees for shelter and bats from one colony only occasionally use trees belonging to the subset of trees used by the other colonies. This structure slows down the spread of diseases and the exchange of information through the entire network. The correlation between network structure and infection dynamics was showed by the use of a simple epidemiological model [Fortuna et al. (2008b)]. We can go further and ask what changes must happen to the spatial dynamics in order to maintain the structure of the roosting network? That is, if some trees disappear, will the colonies reorganize the use of trees in order to best preserve individual colonies' knowledge by minimizing the transfer of information about food resources to outsiders?

Considering instead the spatial network of temporary ponds used by amphibian species [Fortuna et al. (2006)]. Habitat loss experiments, analogous to the removal of species in food webs and plant-animal mutualistic networks, can tell us the importance of keystone patches on the persistence of the species. In the same way, we can estimate where would be the most suitable site to create a new pond in order to most effectively increase the connectivity during, for example, an especially intense dry season. It would also be interesting to explore how the structure of the network influences the dispersal movements of amphibian species. Could particular network topologies connecting the temporary ponds induce changes in the dispersal kernels of amphibians?

The most challenging and promising field for the application of networks of spatial dynamics is within population genetics. We have seen how network approaches can shed light into the characterization of gene flow patterns inside a tree population [Fortuna et al. (2008a)]. We can also investigate the dynamical implications of the patterns of intraspecific genetic variability among populations. These patterns can be well- 

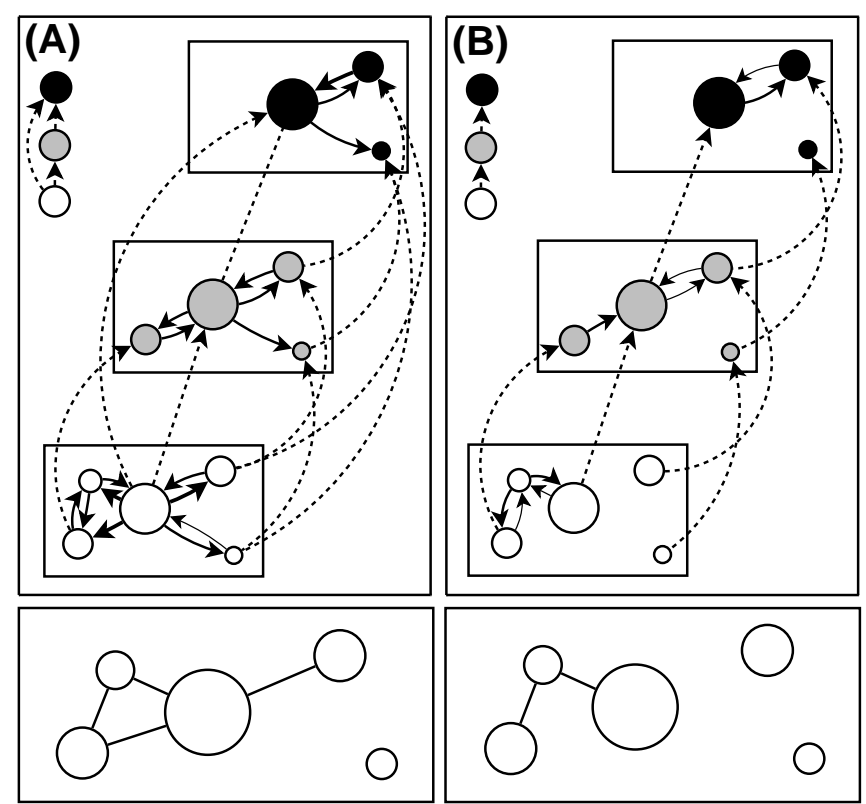

Fig. 1.7 Hypothetical diagram showing the effect of the structure of simple trophic modules such as (A), omnivory and (B), the simple trophic chain on the gene landscape of their constituent species in a spatial network of habitat patches. Each circle represent a patch holding a population. Black, gray, and white patches are occupied by the top, intermediate, and basal species, respectively. Solid arrows represent dispersal movements among populations (i.e., gene flow). Dashed arrows indicate trophic links between species occupying the same patches. Below, the gene landscape of the basal species is represented for both cases. In here, links among patches indicate dependent genetic covariance among them, that is, the holding populations are genetically similar. Isolated patches represent independent evolutionary units (i.e., their genetic covariance is independent of the other populations). If this hypothetical situation is observed in nature, it could imply that more complex networks (omnivory versus simple trophic chain) reduce the genetic structure of populations by maintaining the genetic diversity.

characterized with spatial network approaches, as demonstrated by Dyer and Nason (2004). They described the statistical relationships between all populations simultaneously defining a "population graph" that can also be 
viewed as a "genetic landscape". In a genetic landscape, nodes represent populations and a link indicates a high degree of genetic similarity between them [Dyer and Nason (2004)]. What are the dynamical implications of the topology of the genetic landscape on microevolutionary processes, as these ultimately can lead to a significant evolutionary change? Do the dynamics of communities generate a universal spatial pattern in the distribution of genetic variability between species?

We can go further by coupling the networks of interacting species with the complex spatial context in which they live. The spatial distribution of species is heterogeneous. Different species are therefore found in different habitat patches. We can think about networks of interacting species embedded into the spatial networks of these habitat patches. These represent "meta-webs" which operate on multiple scales. One component of biodiversity depends on local processes, such as interactions among species, and the other component depends on regional processes, such as dispersal between habitat patches. By studying the dynamics of these networks in spatially explicit landscapes, we could integrate population genetics to explore how specific interactions among species shape the structure of gene landscapes (Fig. 1.7).

\subsection{Concluding remarks}

Throughout this chapter, we hoped to leave the reader with at least one indelible idea. The time is now in ecology to stop considering species or patches as independent units but instead as parts of a larger, more complex, network of interacting pieces. The structural properties which describe these interactions - food webs, mutualistic networks, and spatial networks - have begun to take shape, but the understanding of these networks' dynamics is only just beginning. We have provided but a few examples of where work on ecological network dynamics can lead us. Wherever this happens to be, the future is bright, the potential results critical, and the possibilities endless. 


\section{Bibliography}

Albert, R., Jeong, H. and Barabási, A. (2000). Error and attack tolerance of complex networks, Nature 406, 6794, pp. 378-382.

Allesina, S., Alonso, D. and Pascual, M. (2008). A general model for food web structure. Science 320, 5876, pp. 658-661.

Amaral, L. A. N. and Ottino, J. (2004). Complex networks: Augmenting the framework for the study of complex systems, Eur. Phys. J. B 38, 2, pp. $147-162$.

Barabasi, A. and Albert, R. (1999). Emergence of scaling in random networks, Science 286, 5439, pp. 509-512.

Barrett, S. C. H. and Harder, L. D. (1996). Ecology and evolution of plant-mating, Trends Ecol. Evol. 11, pp. 73-79.

Bascompte, J., Jordano, P., Melian, C. J. and Olesen, J. M. (2003). The nested assembly of plant-animal mutualistic networks, Proc. Natl. Acad. Sci. USA 100, 16, pp. 9383-9387.

Bascompte, J., Jordano, P. and Olesen, J. M. (2006). Asymmetric coevolutionary networks facilitate biodiversity maintenance, Science 312, 5772, pp. 431433.

Bascompte, J. and Melián, C. J. (2005). Simple trophic modules for complex food webs, Ecology 86, 11, pp. 2868-2873.

Bascompte, J., Melián, C. J. and Sala, E. (2005). Interaction strength combinations and the overfishing of a marine food web, Proc. Natl. Acad. Sci. U. S. A. 102, 15, pp. 5443-5447.

Bascompte, J. and Solé, R. (eds.) (1998). Modeling Spatiotemporal Dynamics in Ecology (Springer-Verlag, Berlin).

Berlow, E. L. (1999). Strong effects of weak interactions in ecological communities, Nature 398, pp. 330-334.

Berlow, E. L., Neutel, A.-M., Cohen, J. E., de Ruiter, P. C., Ebenman, B., Emmerson, M., Fox, J. W., Jansen, V. A. A., Iwan Jones, J., Kokkoris, G. D., Logofet, D. O., McKane, A. J., Montoya, J. M. and Petchey, O. (2004). Interaction strengths in food webs: issues and opportunities, $J$. Anim. Ecol. 73, 3, pp. 585-598.

Brose, U., Jonsson, T., Berlow, E. L., Warren, P., Banasek-Richter, C., Bersier, 
L.-F., Blanchard, J. L., Brey, T., Carpenter, S. R., Blandenier, M.-F. C., Cushing, L., Dawah, H. A., Dell, T., Edwards, F., Harper-Smith, S., Jacob, U., Ledger, M. E., Martinez, N. D., Memmott, J., Mintenbeck, K., Pinnegar, J. K., Rall, B. C., Rayner, T. S., Reuman, D. C., Ruess, L., Ulrich, W., Williams, R. J., Woodward, G. and Cohen, J. E. (2006). Consumerresource body-size relationships in natural food webs. Ecology 87, 10, pp. $2411-2417$.

Camacho, J., Guimerà, R. and Amaral, L. A. N. (2002). Robust patterns in food web structure, Phys. Rev. Lett. 88, p. art. no. 228102.

Camacho, J., Stouffer, D. B. and Amaral, L. A. N. (2007). Quantitative analysis of the local structure of food webs, J. Theor. Biol. 246, 2, pp. 260-268.

Ceballos, G. and Ehrlich, P. R. (2006). Global mammal distributions, biodiversity hotspots, and conservation, Proc. Natl. Acad. Sci. U. S. A. 103, 51, pp. 19374-19379.

Ceballos, G., Ehrlich, P. R., Soberón, J., Salazar, I. and Fay, J. P. (2005). Global mammal conservation: What must we manage? Science 309, 5734, pp. 603-607.

Chapin, F. S., Zavaleta, E. S., Eviner, V. T., Naylor, R. L., Vitousek, P. M., Reynolds, H. L., Hooper, D. U., Lavorel, S., Sala, O. E., Hobbie, S. E., Mack, M. C. and Díaz, S. (2000). Consequences of changing biodiversity, Nature 405, pp. 234-242.

Cochrane, M. A., Alencar, A., Schulze, M. D., Sourza, C. M., Jr., Depstad, D. C., Lefebvre, P. and Davidson, E. A. (1999). Positive feedbacks in the fire dynamic and closed canopy tropical forests, Science 284, pp. 18321835 .

Cohen, J. E. (1978). Food Webs and Niche Space (Princeton University Press, Princeton, NJ).

Cohen, J. E., Briand, F. and Newman, C. M. (1990). Community Food Webs: Data and Theory (Springer-Verlag, Berlin).

Cohen, J. E., Jonsson, T. and Carpenter, S. R. (2003). Ecological community description using the food web, species abundance, and body size, Proc. Natl. Acad. Sci. U. S. A. 100, pp. 1781-1786.

Dunne, J., Williams, R. and Martinez, N. (2002a). Network structure and biodiversity loss in food webs: robustness increases with connectance, Ecol. Lett. 5, pp. $558-567$.

Dunne, J. A., Williams, R. J. and Martinez, N. D. (2002b). Food-web structure and network theory: The role of connectance and size, Proc. Natl. Acad. Sci. U. S. A. 99, pp. 12917-12922.

Dunne, J. A., Williams, R. J., Martinez, N. D., Wood, R. A. and Erwin, D. H. (2008). Compilation and network analyses of cambrian food webs. PLoS Biol 6, 4, p. e102.

Dyer, R. J. and Nason, J. D. (2004). Population graphs: the graph theoretic shape of genetic structure, Mol. Ecol. 13, pp. 1713-1727.

Ehrlich, P. and Raven, P. (1964). Butterflies and plants: a study in coevolution, Evolution 18, pp. 586-608.

Emmerson, M. C. and Yearsley, J. M. (2004). Weak interactions, omnivory and 
emergent food-web properties, Proc. R. Soc. Lond. B 271, pp. 397-405.

Fortuna, M. A. and Bascompte, J. (2006). Habitat loss and the structure of plantanimal mutualistic networks, Ecol. Lett. 9, pp. 281-286.

Fortuna, M. A., García, C., Guimaraes, P. and Bascompte, J. (2008a). Spatial mating networks in insect-pollinated plants, Ecol. Lett. 11, pp. 490-498.

Fortuna, M. A., Gomez-Rodríguez, C. and Bascompte, J. (2006). Spatial network structure and amphibian persistence in stochastic environments, Proc. $R$. Soc. B. 273, pp. 1429-1434.

Fortuna, M. A., Popa-Lisseanu, A., Ibañez, C. and Bascompte, J. (2008b). The roosting spatial network of a bird-predator bat, Ecology in press.

Fussmann, G. F. and Heber, G. (2002). Food web complexity and chaotic population dynamics, Ecol. Lett. 5, pp. 394-401.

Gibbons, P. and Lindenmayer, D. (2002). The hollows and wildlife conservation in Australia (Csiro Publishing, Australia).

Griffith, D. R. (2008). The ecological implications of individual fishing quotas and harvest cooperatives, Front. Ecol. Environ. 6, 4, pp. 191-198.

Guimarães, P., Jr, Machado, G., de Aguiar, M., Jordano, P., Bascompte, J., Pinheiro, A. and dos Reis, S. (2007). Build-up mechanisms determining the topology of mutualistic networks, J. Theor. Biol. 249, pp. 181-189.

Hansen, M. C., Stehman, S. V., Potapov, P. V., Loveland, T. R., Townshend, J. R. G., Defries, R. S., Pittman, K. W., Arunarwati, B., Stolle, F., Steininger, M. K., Carroll, M. and Dimiceli, C. (2008). Humid tropical forest clearing from 2000 to 2005 quantified by using multitemporal and multiresolution remotely sensed data. Proc. Natl. Acad. Sci. U. S. A. .

Hanski, I. and Gilpin, M. E. (1997). Metapopulation biology: ecology, genetics and evolution (Academic Press).

Heithaus, M. R., Frid, A., Wirsing, A. J. and Worm, B. (2008). Predicting ecological consequences of marine top predator declines, Trends Ecol. Evol. 23, 4, pp. 202-210.

Holt, R. D. (1997). Community modules, in A. C. Gange and V. K. Brown (eds.), Multitrophic Interactions in Terrestrial Ecosystems, 36th Symposium of the British Ecological Society (Blackwell Science, Oxford), pp. 333-349.

Holt, R. D. and Hochberg, M. E. (2001). Indirect interations, community modules and biological control: a theoretical perspective, in E. Waijnberg, J. K. Scott and P. C. Quimby (eds.), Evaluation of Indirect Ecological Effects of Biological Control (CAB International), pp. 13-37.

Holyoak, M., Leibold, M. and Holt, R. (eds.) (2005). Metacommunities: spatial dynamics and ecological communities (Chicago University Press).

Hurlbert, A. H. and Jetz, W. (2007). Species richness, hotspots, and the scale dependence of range maps in ecology and conservation, Proc. Natl. Acad. Sci. U. S. A. 104, 33, pp. 13384-13389.

Jordano, P., Bascompte, J. and Olesen, J. M. (2003). Invariant properties in coevolutionary networks of plant-animal interactions, Ecol. Lett. 6, 1, pp. $69-81$.

Krishna, A., Guimarães, P., Jr, Jordano, P. and Bascompte, J. (2008). A neutralniche theory of nestedness in mutualistic networks, Oikos In press. 
Lawton, J. H. (1989). Food webs, in J. Cherrett (ed.), Ecological Concepts (Blackwell Scientific, Oxford, UK), pp. 43-78.

Leibold, M., Holyoak, M., Mouquet, N., Amarasekare, P., Chase, J., Hoopes, M., Holt, R., Shurin, J., Law, R., Tilman, D., Loreau, M. and Gonzalez, A. (2004). The metacommunity concept: a framework for multi-scale community ecology, Ecol. Lett. 7, pp. 601-613.

McAllister, D., Scheuler, F. W., Roberts, C. M. and Hawkins, J. P. (1994). Mapping and GIS analysis of the global distribution of coral reef fishes on an equal-area grid, in R. I. Miller (ed.), Mapping the Diversity of Nature (Chapman \& Hall, London), pp. 155-175.

McCann, K. S. (2000). The diversity-stability debate, Nature 405, pp. 228-233.

McCann, K. S., Hastings, A. and Huxel, G. R. (1998). Weak trophic interactions and the balance of nature, Nature 395, pp. 794-798.

Melián, C., Bascompte, J. and Jordano, P. (2005). Spatial structure and dynamics in a marine food web, in A. Belgrano, U. Scharler, J. Dunne and R. Ulanowicz (eds.), Aquatic Food Webs, chap. Spatial structure and dynamics in a marine food web (Oxford Univ Press, Oxford), pp. 19-24.

Memmott, J., Waser, N. and Price, M. (2004). Tolerance of pollination networks to species extinctions. Proc. Biol. Sci. 271, 1557, pp. 2605-2611.

Milo, R., Shen-Orr, S., Itzkovitz, S., Kashtan, N., Chklovskii, D. and Alon, U. (2002). Network motifs: Simple building blocks of complex networks, Science 298, 5594, pp. 824-827.

Minor, E. S. and Urban, D. L. (2008). A graph-theory framework for evaluating landscape connectivity and conservation planning, Conserv. Biol. 22, pp. 297-307.

Myers, N., Mittermeier, R. A., Mittermeier, C. G., da Fonseca, G. A. B. and Kent, J. (2000). Biodiversity hotspots for conservation priorities, Nature 403, pp. 853-858.

Myers, R. A. and Worm, B. (2003). Rapid worldwide depletion of predatory fish communities. Nature 423, 6937, pp. 280-283.

Nakazawa, T. and Yamamura, N. (2006). Community structure and stability analysis for intraguild interactions among host, parasitoid, and predator, Popul. Ecol. 48, pp. 139-149.

National Marine Fishery Service (2008). Annual report to Congress on the Status of US Fisheries-2007, Tech. rep., U.S. Department of Commerce, NOAA, Natl., Mar. Fish. Serv., Silver Spring, Maryland.

Nepstad, D. C., Verissimo, A., Alencar, A., Nobre, C., Lima, E., Lefebvre, P., Schlesinger, P., Potter, C., Moutinho, P., Mendoza, E., Cochrane, M. and Brooks, V. (1999). Large-scale impoverishment of Amazonian forests by logging and fire, Nature 398, pp. 505-508.

NERC Centre for Population Biology, I. C. (1999). The Global Population Dynamics Database. http://www.sw.ic.ac.uk/cpb/cpb/gpdd.html.

Olesen, J., Bascompte, J., Elberling, H. and Jordano, P. (2008). Temporal dynamics in a pollination network, Ecology 89, pp. 1573-1582.

Otto, S. B., Rall, B. C. and Brose, U. (2007). Allometric degree distributions facilitate food-web stability. Nature 450, 7173, pp. 1226-1229. 
Ovaskainen, O. and Hanski, I. (2004). Ecology, genetics and evolution of metapopulations, chap. Metapopulation dynamics in highly fragmented landscapes (Academic Press), pp. 73-104.

Paine, R. T. (1988). Food webs: Road maps of interactions or the grist for theoretical development? Ecology 69, 6, pp. 1648-1654.

Pascual, M. and Dunne, J. A. (eds.) (2006). Ecological Networks: Linking Structure to Dynamics in Food Webs (Oxford University Press, Oxford, UK).

Petanidou, T., Kallimanis, A., Tzanopoulos, J., Sgardelis, S. and Pantis, J. (2008). Long-term observation of a pollination network: fluctuation in species and interactions, relative invariance of network structure and implications for estimates of specialization, Ecol. Lett. 11, pp. 564-575.

Petchey, O. L., Beckerman, A. P., Riede, J. O. and Warren, P. H. (2008). Size, foraging, and food web structure. Proc. Natl. Acad. Sci. U. S. A. 105, 11, pp. 4191-4196, doi:10.1073/pnas.0710672105.

Pimm, S. L. (2002). Food webs, 1st edn. (University of Chicago Press, Chicago, IL).

Pimm, S. L. and Raven, P. (2000). Extinction by numbers, Nature 403, pp. 843-845.

Rezende, E., Jordano, P. and Bascompte, J. (2007a). Effects of phenotypic complementarity and phylogeny on the nested structure of mutualistic networks, Oikos 116, pp. 1919-1929.

Rezende, E., Lavabre, J., Guimarães, P., Jr, Jordano, P. and Bascompte, J. (2007b). Non-random coextinctions in phylogenetically structured mutualistic networks, Nature 448, pp. 925-928.

Rooney, N., McCann, K., Gellner, G. and Moore, J. C. (2006). Structural assymetry and the stability of diverse food webs, Nature 442, pp. 265-269.

Santamaría, L. and Rodríguez-Gironés, M. (2007). Linkage rules for plantpollinator networks: trait complementarity or exploitation barriers? PLoS Biol 5(2), p. e31.d0i:10.

Schick, R. S. and Lindley, S. (2007). Directed connectivity among fish populations in a riverine network, J. Appl. Ecol. 44, pp. 1116-1126.

Skole, D. and Tucker, C. J. (1993). Evidence for tropical deforestation, fragmented habitat, and adversely affected habitat in the Brazilian Amazon:1978-1988, Science 260, pp. 1905-1910.

Solé, R. and Montoya, J. (2001). Complexity and fragility in ecological networks. Proc. Biol. Sci. 268, 1480, pp. 2039-202020202020202020202020202020202020202045.

Stang, M., Klinkhamer, G., Peter and van der Meijden, E. (2007). Asymmetric specialization and extinction risk in plant-flower visitor webs: a matter of morphology or abundance? Oecologia 151, pp. 442-453.

Stouffer, D. B., Camacho, J. and Amaral, L. A. N. (2006). A robust measure of food web intervality, Proc. Natl. Acad. Sci. U. S. A. 103, 50, pp. 1901519020.

Stouffer, D. B., Camacho, J., Guimerà, R., Ng, C. A. and Amaral, L. A. N. (2005). Quantitative patterns in the structure of model and empirical food webs, Ecology 86, pp. 1301-1311. 
Stouffer, D. B., Camacho, J., Jiang, W. and Amaral, L. A. N. (2007). Evidence for the existence of a robust pattern of prey selection in food webs, Proc. R. Soc. B 274, 1621, pp. 1931-1940.

Sugihara, G. (1984). Graph theory, homology, and food webs, in S. A. Levin (ed.), Population Biology, Proceedings of Symposia in Applied Mathematics, Vol. 30 (American Mathematical Society), pp. 83-101.

Thomas, J. A., Telfer, M. G., Roy, D. B., Preston, C. D., Greenwood, J. J. D., Asher, J., Fox, R., Clarke, R. T. and Lawton, J. H. (2004). Comparative losses of British butterflies, birds, and plants and the global extinction crisis, Science 303, pp. 1879-1881.

Thompson, J. N. (2005). The Geographic Mosaic of Coevolution (University of Chicago Press, Chicago, IL, USA).

Tilman, D. and Kareiva, P. (1997). Spatial ecology: the role of space in population dynamics and interespecific interactions (Princeton University Press).

Tylianakis, J. M., Tacharntke, T. and Lewis, O. T. (2007). Habitat modification alters the structure of tropical host-parasitoid food webs, Nature 445, pp. 202-205.

United Nations Food and Agriculture Organization (2006). The State of World Fisheries and Aquaculture, Tech. rep., United Nations, Rome, Italy.

Urban, D. L. and Keitt, T. (2001). Landscape connectivity: a graph-theoretic perspective, Ecology 82, pp. 1205-1218.

Vázquez, D. P. and Aizen, M. A. (2004). Asymmetric specialization: a pervasive feature of plant-pollinator interactions, Ecology 85, pp. 1251-1257.

Waser, N., Chittka, L., Price, M., Williams, N. and Ollerton, J. (1996). Generalization in pollination systems, and why it matters, Ecology 77, 4, pp. $1043-1060$.

Wiens, J. A. (2001). Dispersal, chap. The landscape context of dispersal (Oxford University Press), pp. 96-109.

Williams, R. J. and Martinez, N. D. (2000). Simple rules yield complex food webs, Nature 404, pp. 180-183.

Williams, R. J. and Martinez, N. D. (2008). Success and its limits among structural models of complex food webs, Journal of Animal Ecology 77, 3, pp. $512-519$.

Worm, B., Sandow, M., Oschlies, A., Lotze, H. K. and Myers, R. A. (2005). Global patterns of predator diversity in the open oceans. Science 309, 5739, pp. $1365-1369$.

Yodzis, P. and Innes, S. (1992). Body size and consumer-resource dynamics, American Naturalist 139, pp. 1151-1175. 